\title{
Supporting Emergency Medicine Research: Developing the Infrastructure
}

\author{
Michelle H. Biros, MS, MD, William G. Barsan, MD, Roger J. Lewis, MD, PhD,
} Arthur B. Sanders, $M D$

\begin{abstract}
The long-term goals of developing research within the specialty of emergency medicine (EM) include the following: 1) to continue to improve the quality and quantity of EM research in order to ultimately improve emergency patient care; 2) to maximize the research potential of emergency health care professionals in order to develop new emergency research talent and enthusiasm; and 3) to establish the academic research credentials of the specialty of EM in order to become competitive for federal research funding, and further improve emergency patient care. This article addresses the process by which the infrastructure for EM research can be developed at academic medical centers and provides recommendations. The roles of the academic chair, research director, senior researcher, and departmental faculty are discussed.
\end{abstract}

Key words: emergency medicine research; departmental research support; research infrastructure.

Acad. Emerg. Med. 1998; 5:177-184.

- The specialty of emergency medicine (EM) came into existence to meet a clinical need in the health care system. As the specialty grew, its core content, knowledge base, and skills list quickly developed. This was accompanied by the establishment of training programs to ensure competency in the clinical practice of EM. Research and academic development of EM followed from the underlying clinical discipline. Because of the newness of the specialty, traditional academic lines of advancement were not available for those interested in academic EM. These traditions had to be created as the specialty grew and gained legitimacy in the house of medicine, especially at university medical centers. The past 15 years has witnessed a remarkable growth in both the quality and the quantity of research in EM. The purpose of this report is to discuss issues that must be addressed to enhance support for basic, clinical, and health services research in EM at the local and national levels.

Long-term goals of developing research within the specialty of EM include the following: 1) to continue to improve the quality and quantity of EM research in order to ultimately improve emergency patient care; 2 ) to maximize the research potential of emergency health care professionals

From Hennepin County Medical Center, Minneapolis MN, Department of Emergency Medicine (MHB); University of Michigan, Ann Arbor, MI, Section of Emergency Medicine (WGB); Harbor-UCLA Medical Center, Torrance, CA, Department of Emergency Medicine (RJL); and University of Arizona Medical Center, Tucson, AZ, Division of Emergency Medicine (ABS).

Received: August 15, 1997; accepted: August 27, 1997.

Prior presentation: The Future of Emergency Medicine Research Conference, Washington, DC, March 1997.

Address for correspondence and reprints: Michelle H. Biros, MS, MD, Department of Emergency Medicine, Hennepin County Medical Center, 701 Park Avenue South, Minneapolis, MN 55415. Fax:612-904-4241; e-mail: michelle.biros@co.hennepin.mn.us

This article is being copublished in Academic Emergency Medicine and the Annals of Emergency Medicine. (O1998 Hanley \& Belfus, Inc.

in order to develop new emergency. research talent and enthusiasm; and 3) to establish the academic research credentials of the specialty of EM in order to become competitive for federal research funding, and further improve emergency patient care.

These goals can be achieved by fostering a supportive environment and culture in which research is nurtured and valued at the departmental, institutional, regional, and national levels. Good research programs require a significant investment and commitment to build an infrastructure and culture that will contribute new meaningful medical knowledge.

We believe emergency research is necessary for the continued development of the specialty of EM and the improvement of patient care. Thus, research needs to be supported by the specialty as well as by individual institutions as an essential part of the clinical discipline of EM. Once it is part of the local and specialty infrastructure, research productivity becomes an accepted and expected part of the academic mission of EM training programs. The issue of research goes beyond the individual researchers themselves or even beyond academicians in EM. It is an issue that 
the specialty must promote and support as essential for the specialty's academic growth and development, and its commitment to the continuous improvement of clinical care.

\section{OBSTACLES TO PERFORMING EM RESEARCH}

An important challenge for the specialty of EM will be to foster and continue the growth and development of its research base in a time of continued financial pressures and cutbacks of research and institutional resources. To do this, research must be transformed into an important part of the professional culture of EM. Research should not be considered something separate or expendable from the specialty. Instead, emergency research must be recognized as essential to the advancement of clinical EM. Specific issues that must be addressed to successfully advance our academic goals include protected time for research, mentorship, research training and experience, research facilities, institutional support, and the variabilities in research environments between institutions.

Protected time for researchers to develop skills in their area of interest and to allow networking with authorities in their research field is essential for the development of good research. EM has an important clinical base with responsibilities for staffing emergency departments (EDs) 24 hours a days, 365 days a year. This heavy clinical responsibility leads to intense pressures on those interested in academic EM. EM academicians must be clinically productive and, in most cases, generate salary support for themselves through clinical activities. They also must be academically productive and responsive to demands to perform, publish, and selffund research endeavors. Many disciplines have considerably less clinical responsibility, allowing increased protected time for young faculty as they develop their research base.
In some other medical specialties, prospective faculty are expected to complete research training fellowships before joining an academic department. Thus, academic faculty may have spent 2-3 years after specialty training developing their own areas of research expertise, and developing the skills and knowledge needed to compete for grant support. Part of this research training involves socialization and learning how to survive in an academic environment. Fellowship training in EM has expanded greatly over the past decade. Many EM fellowships, however, are based in specific clinical areas such as toxicology and emergency medical services. There are only a few fellowships that provide the extensive training in advanced research concepts and methodology that is needed to successfully compete on a national level for the limited financial resources allocated to research.

Because of the newness of the discipline of EM, lines of academic advancement and mentors for research in EM are few. Many leaders in EM have arisen because of their political or clinical skills, rather than by fulfilling traditional academic criteria, which heavily emphasize academic accomplishments. Because their exposure to research may be limited, some department chairs are not familiar with efforts required to produce credible research. Even faculty who have completed fellowship training and have tremendous potential for independent research may be put in positions without the necessary financial support and protected time to develop a meaningful research career. Chiefs may also prematurely appoint a new faculty member with research fellowship training or previous research experience to the position of "research director." This faculty member is then expected not only to develop his or her own research base, but also to help other faculty, residents, and fellows produce research in other areas. The time management, administrative, and facilitating skills necessary to perform this function make this position appropriate for a more senior faculty member.

In many other disciplines, a variety of research mentors are available to encourage and stimulate faculty development. In EM, mentors often need to be sought out. Novice researchers should be encouraged to seek out mentors in other disciplines, if none are available at their institution within academic EM.

Each institution is unique, and the development of a successful research effort will be distinct for every institution. Scholarly research can be performed in many settings and the breadth of research in EM is a strong advantage of the discipline. Thus, advancing research at the institutional level will come from working within the local environment. While all areas of the broad scope of EM research, including basic science, clinical science, and health services, will not be done at every institution, each institution should be involved in more than one area of research. This diversification allows for intellectual stimulation and internal support by researchers with different foci in the same department. The breadth of EM research allows faculty to develop many different specific areas and collaborations in local institutions. Furthermore, effective EM clinical investigation generally requires the collaboration of many clinicians from within and outside of the ED.

While more than one area of research activity may promote the immediate development of the departmental's research base, too many paths of research may retard the longterm growth process. Academic EDs and investigators need to establish credibility in their field of research interest. This can be done only by establishing a history of productivity and visibility in a particular area of research. Identification as a noted expert in a particular research area enhances the likelihood of successful competition for scarce financial resources, invitations for membership 
on national research review boards or panels, and participation in federal grant review processes. Such activities are essential for establishing EM as a competitive academic specialty with credentials as impressive as those of disciplines with a longer history of academic productivity. The more the unique characteristics of a department's clinical and basic science research setting complements the specific interests of the department's researchers, the easier it will be to create an active productive research base.

An academic department should develop more than one researcher within each area of research interest. A critical mass of enthusiastic investigators is needed to enhance dialogue, generate resources, and increase networking within and outside of the department. If an academic program relies on 1 or 2 individuals for its research productivity, the program may collapse if these individuals leave or change directions.

\section{OVERCOMING THE OBSTACLES}

\section{The Chief's Role:}

Departmental Issues. Although most research performed in a department will not be directly done by the chair or chief, the chief's role is critical in developing the environment in which productive research can occur. The ability to perform research and successfully compete for extramural funding typically will require an initial infusion of capital, in the form of equipment costs, animal costs, personnel costs, and/or release time. As the individual usually responsible for the purse strings, the chief can profoundly affect the ability of a department to be successful in this area. The chief's role is particularly important in establishing an environment conducive to productive research, setting clear expectations for faculty members with regard to research, and directing the financial support of research endeavors.

\section{RECOMMENDATIONS}

1. The chief should create an environment in which research is not merely tolerated, but is actively promoted. The development of a research environment starts with the chief's own vision and will be reflected in the recruitment of other faculty members. It should be clearly understood that a major departmental goal is production of meaningful research and procurement of extramural funding. It should further be understood that this will require diversion of funds that might otherwise be available for salary support. All faculty members must buy into the departmental goals for research. These goals may be specified in terms of the expected amount of extramural funding obtained, number of research publications, national research presentations, etc. Departmental research goals will be easier to achieve if all department members understand what they are. A specific plan for designating a certain percentage of the yearly net revenue to research should be developed. agreed to, and understood by all faculty members in the department. Likewise, a specific expectation for research productivity should highlight the effectiveness of the department's commitment to and involvement in the research effort.

\section{A specific plan for academic re-} search development should be created with clearly defined goals and expectations. Besides creating the environment in which research is promoted, the chief can play an important role in providing direction, goals, and time for academic research faculty. The chief's academic development plan should advise the research faculty in effective time management, realistic expectations for yearly productivity, and appropriate mentorship.

Mentorship may be provided by individuals within the department of EM, or by individuals from other departments. The chief should assist in identifying an appropriate mentor, and establishing a working relation- ship between the mentor and the research faculty. Mentors provide advice and information on basic research issues such as methodology and funding sources. Mentors can facilitate research efforts of novice investigators because of their greater experience and understanding of the local and national research environment. A good mentor provides an altruistic, visionary role model for the developing researcher. The selection of an appropriate mentor can be a crucial step in the initiation of a successful research career.

3. The chief should provide start-up funds for preliminary work. Individual researchers will typically require some amount of up-front (or seed) money to enable them to develop their research to the point where they are capable of applying for extramural funds. The monetary investment is similar to start-up funds for a new business. Extramural funding agencies will not seriously consider any research proposal that does not include a significant amount of preliminary work. Besides allocating a designated amount of start-up money, the chief also must provide an appropriate physical setting for research activity. This may mean outfitting a laboratory or arranging for shared space in other laboratories. This again will usually require a financial outlay.

4. The chief must provide research faculty with protected nonclinical research time. Typically, meaningful research cannot be accomplished if a faculty member is working more than 24 clinical hours per week. ${ }^{1}$ Having part-time faculty members to fill in for clinical shifts for research faculty is one option for providing protected time. The chief should also ensure that the researcher's nonclinical time is not consumed by administrative responsibilities such as chairing numerous committees, being in charge of EMS, etc. These activities would defeat the purpose of "protected" time, and should be assigned to non-re- 
search faculty who are also being given adequate time to perform these responsibilities. On the other hand, the chief should attempt to demonstrate the knowledge, organizational talents, and research enthusiasm of EM researchers to the institutional research community. Appointment to key institutional committees [institutional review board (IRB), the animal research committee, research, education, intramural grant review, etc.] not only establishes an EM research presence but also introduces EM researchers to institutional colleagues who may eventually participate in decisions regarding their academic promotion.

\section{The chief should attempt to recruit} the researchers of tomorrow. Meaningful research opportunities should be available for interested medical students and undergraduate students. Students who show an interest in research can thus be encouraged to pursue a career in EM and potentially contribute to our future research. Likewise, research interests and accomplishments of prospective residents should be considered strongly in the resident selection process.

Institutional Issues. It is critical for EM to develop a credible research stature in academic institutions. Gaining acceptance in most academic institutions necessitates a significant emphasis on research activities. The departmental chief not only must be supportive of research activities in his or her department, but also must demonstrate this concern institutionally.

\section{RECOMMENDATIONS}

1. The chief should secure an institutional commitment to the department's research efforts. The earliest point at which this commitment can be illustrated is during the recruitment process for a new chief. As part of the recruitment package, candidates for the chief's position should typically ask for specific support for research activities. At the least, these requests should include adequate laboratory space for research activities, adequate salary support to ensure recruitment and retention of high-quality faculty, adequate nonclinical time to conduct research, a significant amount of discretionary funds, which can be used to foster research efforts, seed money for all new research faculty members, support for technical personnel for research, and institutional help in recruiting PhD researchers. The best time to secure such an institutional commitment is during negotiations for a new position rather than after the contract has been signed.

2. Collaborative efforts with researchers in other departments should be encouraged and promoted. Collaborative research should be actively promoted for researchers at all levels. Within an institution, emergency academicians have a "tendency towards academic isolation." 2 Due to clinical hours that do not necessarily correspond to those of other institutional researchers or research resources, many emergency physicians do not develop the special skills needed to engage in collaborative multidisciplinary research. As suggested by the distribution of Emergency Medicine Foundation (EMF) advanced research grant awards, many emergency researchers have successfully engaged in collaborative endeavors with basic scientists. ${ }^{3}$ Similar successful collaboration with established clinical researchers is also essential since many novice emergency researchers have little formal training or experience in the research methodologies of clinical trials.

In order to establish collaborations, institutional researchers must be made aware of the department's efforts. EM research productivity should be publicized and promoted. Researchers from other departments should be invited to present their research findings to the department of EM, and the reciprocal opportunity to present EM research to other departments should be sought. EM should be represented in institutional research conferences, paper presentations, and seminars on grant funding. The EM department should try to identify the availability of shared equipment and space in other department laboratories, and make equipment in the EM laboratories available for use by others. Research expertise in other departments should be used. In clinical research, it is crucial that EM be actively involved in any research that involves ED patients. Investigators from other departments who desire to do EM clinical research should be required to formally collaborate with an EM research faculty. We would not try to do research on intensive care unit (ICU) patients without having the "buy-in" of the ICU team, and in the same way other physicians should not assume they can do research involving ED patients without having the "buy-in" of ED faculty. This involvement is necessary to define our research environment. It also ensures an adequate and accurate interpretation of ED data, allows scientific and ethical scrutiny of a project to be done involving our patients, and provides input into the feasibility and the possible limitations of data collection in our practice setting.

Residents can also benefit from research collaboration with other departments. Residents from other specialties can be invited into the department's research laboratories or clinical research settings, and arrangements can be made to allow EM residents to work in the laboratories or clinical setting of another department. Such sharing of knowledge and resources helps to bring EM more solidly into the institutional research community.

\section{The ED should be represented on} key institutional research committees. A potential stumbling block for implementation of many emergency research protocols occurs when those protocols are reviewed by various research committees with no EM perspective. For instance, the IRB mem- 
bership often has little familiarity with standard EM practice, making it difficult for them to judge the appropriateness or safety of proposed research protocols. An EM researcher is an important information source, and can provide the IRB with the background needed to fairly evaluate the quality of the proposal in question.

The Role of Clinical Colleagues: In addition to the support of research established by the department chief, non-researcher clinical colleagues can further support the department researcher by promoting research endeavors.

\section{RECOMMENDATIONS}

1. Clinical EM colleagues should assist in identification and enrollment of appropriate ED patients for clinical research. Clinical colleagues should remind residents, students, and other staff of current departmental research efforts, and actively recruit potential research subjects. This will increase the likelihood that a research project will be completed in a timely fashion and will stimulate continued research interest among all department members.

2. Clinical EM colleagues should provide an objective appraisal of a research proposal. The practical input from non-researchers on a proposed research study may indicate the need to clarify or modify some aspects of the study design. A constructive critique is also useful for research abstracts, grant applications, or manuscripts. This critique allows improvement of clarity in the written word, may challenge the feasibility of a particular project in the local research environment, may provide additional ideas regarding research collaboration, and may offer new insights into the practical completion or interpretation of the study. Non-researcher departmental colleagues can offer this type of information and objective criticism regarding research proposals and results.
Research Directors: In many EM departments, one of the faculty members is designated as the "research director." This individual is usually appointed by the chief to administer the research efforts of the department. The role of the research director is determined by the research sophistication of the faculty and the chief. Not all EM departments need a designated research director. In some departments with long-standing histories of research productivity, most of the academic faculty have considerable research experience and are comfortably able to initiate, execute, analyze, and present their research efforts. Senior research faculty divide the department's research teaching and administration, while aggressively continuing their own research efforts. Policies and procedures for conducting departmental research are developed by the group and mutually agreed upon. Researchers from other departments can identify or are referred to the most appropriate person for potential collaboration. Each EM researcher has the knowledge of the local research environment needed to develop his or her own research initiative.

In other EM departments, the research effort is relatively new, and most faculty and the chief may have little previous research experience. In these circumstances, the research director may be instrumental in developing a solid research base. While the appointment of a research director may do much to increase the research productivity of the department, the responsibility of ensuring academic development continues to lie with the chief.

The activities of a departmental research director may be divided into 3 broad categories: 1) educational tasks, where the goal is to improve the research-related knowledge base and skills of faculty and residents within the ED; 2) advisory tasks, where the goal is to give research-related advice regarding specific proposed or ongoing research projects; and 3) facilitation, a broad category of activities aimed at making the conduct of productive research easier for all members of the EM department. The broad scope of these activities makes the research director's role very expansive. The research director may therefore become involved to varying degrees in the majority of the department's research activities. Most research directors do not hesitate to offer assistance when new researchers are developing a proj-ect or floundering in its execution. While this may provide the needed guidance to initiate a department's research program, it must be recognized that this also detracts from the time the research director has available to maintain his or her own area of research expertise.

\section{RECOMMENDATIONS}

1. Research directors should set limits to their involvement in the overall research activity of the department. It is usually not necessary or educationally beneficial for research directors to be involved (and by implication, responsible for) the day-to-day execution of all research projects. Instead, the research director should educate, advise, and facilitate as needed. As the department's research efforts come to fruition, and as the specialty develops research notability and a cadre of sophisticated researchers, the role of research directors should change and become more passive.

\section{The research director should pro-} vide instruction on basic research concepts, or make appropriate referrals to other investigators or pertinent written material. The research director is frequently called upon to teach other faculty members the skills required for the successful completion of research projects. While these teaching activities may take the form of formal lectures or seminars, most commonly the research director engages in one-on-one instruction, focusing on specific projects that the novice investigator is initiating. New investigators often need instruction 
on the development of a research protocol, the performance of sample-size calculations, the preparation of materials for submission to the local IRB or animal use committees, the development of data forms, the organization of electronic databases, the planning of statistical analyses, and the analysis and presentation of results. ${ }^{4}$ More advanced investigators may need instruction in grant preparation and in revising and improving grant applications and manuscripts. ${ }^{5}$ If the research director cannot provide this necessary information directly, he or she should be able to direct the novice investigator to an appropriate source.

\section{The research director should serve} as a source of information regarding local and national research conferences, funding opportunities appropriate for the research being conducted in the department, and other research-related issues. Many academic EDs are affiliated with universities that, in support of their own research or graduate programs, conduct seminars on grant preparation, scientific writing, and other related topics. This information is also useful to novice researchers, and should be made available to them.

4. The research director is responsible for maintaining high departmental standards for the ethical and professional conduct of research. These standards are reflected in the types of research that is conducted, the completeness and accuracy with which applications and IRB submissions are completed, the manner in which patients are recruited and consented for participation in clinical studies, and the manner in which data are analyzed and results presented. In addition to demonstrating the ethical principles of research by example, the research director should include formal teaching of these principles with the instruction of more traditional research concepts. ${ }^{6}$

5. The research director is responsi- ble for familiarizing new investigators with the regulations and local administrative requirements that apply to their research projects. Many new investigators attempt to initiate research projects without a knowledge of institutional and federal regulations that apply to animal and human subjects research. In addition, most institutions have administrative policies and procedures in place to ensure that the research conducted is ethical, is of high quality, and conforms to local and federal regulations. The research director should ensure that all new investigators are familiar with these regulations, policies, and procedures.

6. The research director should assist the investigator in a feasibility assessment of the proposed project, prior to its initiation. One of the most difficult tasks for the novice investigator is the development of a research protocol that addresses a potentially important but intractable problem. A research feasibility assessment includes a realistic appraisal of the planned sample size, the time available to the investigator, the requisite skills and experience, and institutional resources. ${ }^{7}$ A few words of advice or redirection at the earliest stages in the development of a research proposal can save the novice investigator much wasted effort and frustration, and ensure the development of a high-quality protocol.

\section{The research director must ensure} that departmental and extradepartmental resources are fairly allocated to investigators based on need and merit. If the implementation of research projects involves the assistance of residents and students, the research director should ensure that this scarce resource is allocated fairly to different investigators in the department, and that the residents and students derive significant benefits from and credit for their participation in the research. Likewise, the research director should coordinate other re- search resources (i.e., laboratory space, equipment, computer time) so that all departmental research efforts are fairly supported.

8. The research director should assist in the development of networks of consultants and potential collaborators. The successful implementation of many research protocols requires collaboration among members of different departments. Novice investigators need assistance in developing realistic expectations for the contribution of these consultants and collaborators, and the research director can assist with this. Likewise, the research director should direct extradepartmental investigators to appropriate members of the EM faculty to serve as consultants or collaborators. Because new investigators may not be aware of the potential complexity of collaborative research agreements, the research director should develop departmental guidelines outlining the considerations in such collaborations. These guidelines should detail requirements for financial reimbursement, authorship, access to primary data, publication rights, arbitration of disagreements among investigators, etc. The goal of these guidelines is to ensure that departmental investigators, when involved in collaborative efforts, receive fair recognition, credit, and reimbursement for their efforts and, when appropriate, have access to the data that they help to collect.

9. The research director should direct the development of departmental policies for dealing with alleged misconduct, including procedures for the investigation of allegations. Willful misconduct in medical research is relatively infrequent, but has extremely serious implications. The research director should be familiar with the institutional policies for the investigation of allegations of misconduct. If an institutional committee for investigation of scientific misconduct does not exist, it is especially important that the research director be familiar 
with the increasing volume of work published on the definition, detection, and investigation of scientific misconduct. Departmental guidelines for responsible mentorship and authorship should be developed.

The Specialty's Role: Over the last decade, a number of EM departments have developed effective ongoing research programs concentrating on a particular area of emergency research. These programs have been very productive, are visible within our specialty as well as within other specialties with overlapping research and clinical interest, and have become highly competitive for federal and other grant awards. Contributing to this success are institutional support for the EM research operation, departmental success at defining and establishing active interdisciplinary collaboration, active involvement in all phases of project development, and a deep commitment to recruit and retain faculty committed to research. In addition, many of these departments are involved in preclinical (i.e., laboratory-based) research and have ongoing collaborative interactions with non-medically based research specialties (e.g., epidemiology).

\section{RECOMMENDATIONS}

1. Emergency medicine should promote and provide funding for the continued development of recognized successful EM research centers. These established EM research programs are in a unique position to contribute to the research growth of the specialty. By learning from their experience and modeling research program development after their successful operations, other departments may enhance their opportunity to develop similar research success. These programs are also an excellent resource for the education of current and future department chiefs in the importance, needs, and demands of a concerted EM research effort. They can educate research directors and other facilitators in areas of administration, over- sight, collaboration, negotiation, and other skills needed for the development of a successful research enterprise. Existing successful programs are ideal sites for teaching research practices and principles to novice investigators. Obviously, this education is usually directed toward investigators from the program itself, but could also be extended to include novice investigators from other institutions.

2. The specialty should identify and acknowledge sites where exceptional research expertise is available for intellectual exchange. Many productive departments may have a less formalized research organization than those recognized as excellent research centers, but may have developed specialized research models or techniques that might prove useful to an investigator from another institution. The development of "interdepartmental exchange programs" could provide necessary technical training or method sharing, thus advancing the development of potential researchers within our specialty. A lucrative benefit of such shared knowledge is the potential of future collaborative efforts, and the establishment of cordial and long-lasting research relationships with other institutions.

3. The specialty should promote formal research training. EM has been supportive of the development of "subspecialty" fellowships (e.g., toxicology). These fellowships have defined curricula, and the benefits attached to completion are tangible in terms of securing jobs and recognition. The curriculum for general training in research methods is less well described and is variable from institution to institution. In addition, the benefits of general research training fellowships are perceived as nebulous; even current research directors sometimes obtain their jobs with little or no formal training in research principles and practice. While on-the-job training will develop some research skills, it is not controlled, comprehen- sive, or time- or cost-effective. This will become increasingly more challenging as economic pressures impact the academic pursuits of our specialty.

Research training fellowships are best offered at departments that can provide the mentorship necessary to build a solid foundation on research methods, principles, and practice. The continued development of EM research training fellowships should be promoted by the specialty. Likewise, pertinent fellowship training outside of EM (e.g., public health, epidemiology, informatics) should be encouraged to enhance the ultimate research knowledge base of our specialty.

Adequate training in research principles and practice is time-consuming. While a number of short courses have evolved within the specialty (e.g., SAEM Fundamentals of Research, EMF/ACEP Emergency Medicine Basic Research Skills Workshop), it is unrealistic to assume that these short courses provide the skills needed for competitive research endeavors. The value of short courses is to introduce basic research concepts and principles and to begin the evolution of an appropriate research culture for our specialty. However, our specialty must recognize that adequate research training requires a substantial time commitment, and be willing to provide the financial and other resources that are necessary to achieve these ends.

\section{Emergency medicine should sup-} port its own research agenda on all fronts. Our research domain may be clear to us, but it is not widely understood by other specialties and federal funding organizations. Emergency researchers who have developed a subspecialty area of research interest should attend and present research at relevant conferences outside our specialty, and should encourage non-EM researchers to attend and present at our conferences. The specialty should encourage and recognize this intellectual exchange. EM 
must promote its own expertise. Whenever possible, EM should nominate its own researchers for national consensus panels, NIH study sections, safety and data monitoring committees or any other research-related national position that becomes known to us. Not only will this enhance our visibility to other specialities, it will also increase our contacts to the research community at large.

5. Academic EM should develop and constantly reassess current researchrelated policy statements. The best interest of our research development requires that others know where EM stands and how we respond to many sensitive and ethical issues related to the performance of EM research. Policy statements should be developed dealing with the ethical issues of emergency, acute resuscitation, and critical care research. These include, but are not limited to: 1) conflict of interest, 2) dealing with suspected scientific misconduct and fraud, 3) responsible authorship and rules of authorship, and 4) ethical considerations in the performance of industry-sponsored research. These policy statements will not only describe our research principles to others, but will provide guidelines for established EM researchers and excellent training for novice researchers in the "nonscientific" aspects of the research enterprise.

6. The specialty of EM should take a proactive role in national issues of importance regarding acute resusci- tation and critical care research. Established leadership and acknowledged expertise in such issues as the regulation of emergency human subjects research will advance the academic credibility of the specialty within the biomedical research community, and engender public support of EM research.

\section{CONCLUSION}

The development of the infrastructure for EM research is a complex and challenging task that must take place at the departmental, institutional, specialty, and interspecialty levels. Degrees of research sophistication, research educational needs, institutional resources, and the local research environments are different for all EM academic departments.

The final configuration of a department's research organization depends on utilization and supplementation of existing talent, knowledge, and resources. Once it has been established that research is part of the academic mission of the department, the research program can be developed and supported by all faculty members.

The departmental chief has access to financial and institutional resources needed to provide direction to development of the EM research program. By emphasizing the value of research to his or her faculty, the chief will advance the departmental research enterprise.

In some circumstances, a departmental research director can enhance the research knowledge base of departmental researchers. Educational, advisory, and facilitatory tasks are included in the research director's role. As the department's researchers mature, the role of the research director will change.

There are several methods by which the specialty of EM can promote the development of EM research. These include identification and prioritization of research needs, publicizing existing talent and resources, and capitalizing on potential opportunities to advance the reputation of academic EM research and its researchers.

\section{REFERENCES}

1. Meislin HW, Spaite DW, Valenzuela TD. Meeting the goals of academia: characteristics of emergency medicine faculty academic work styles. Ann Emerg Med. 1992; 21:298-302.

2. Petersdorf RG. The place of emergency medicine in the academic community. Ann Emerg Med. 1992; 21:193-200.

3. Rorrie CC. EMF Research Grants Award Listing 1986-1996. Dallas, TX: Emergency Medicine Foundation, 1996.

4. Cline D, Henneman P, Van Ligten P, et al. A model research curriculum for emergency medicine. Ann Emerg Med. 1992; 21:184-92. 5. Baldwin CD, Goldblum RM, Rassin DK, Levine HG. Facilitating faculty development and research through critical review of grant proposals and articles. Acad Med. 1994; 69: $62-4$.

6. Krulwich TA, Friedman PJ. Integrity in the education of researchers. Acad Med. 1993; 68: 814-8.

7. Cummings SR, Brownen WS, Hulley SB. Conceiving the research question. In: Hulley SB, Cummings SR (eds). Designing Clinical Research. Baltimore, MD: Williams and Wilkins, 1988. 